\title{
Der Einfluss staatlicher Vorgaben auf Raumzuschnitt und Institutionalisierung in Regionsbildungsprozessen
}

\author{
Christian Diller ${ }^{1}$ Guido Nischwitz ${ }^{2}$ \\ Eingegangen: 9. November 2020 / Überarbeitet: 19. März 2021 / Angenommen: 22. März 2021 / Online publiziert: 8. April 2021 \\ (c) Der/die Autor(en) 2021
}

\section{Zusammenfassung}

In dem Beitrag geht es um die Frage, welchen Einfluss staatliche Vorgaben auf Prozesse der Regionsbildung haben, insbesondere auf den räumlichen Zuschnitt und die Institutionalisierungsform der Regionen. Die bundesweite empirische Erhebung von 1350 Programmregionen, die aus 27 Programmen von verschiedenen Politikfeldern gefördert wurden, macht zweierlei deutlich: Zum einen können Regionszuschnitt und Institutionalisierung als sich ergänzende Möglichkeiten der stabileren regionalen Zusammenarbeit gesehen werden. Zum anderen werden Regionszuschnitt und Institutionalisierung maßgeblich auch von den staatlichen Vorgaben bestimmt.

Schlüsselwörter Regional Governance $\cdot$ Rescaling $\cdot$ Institutionalisierung $\cdot$ Regionsbildung $\cdot$ Regionale Kooperationen in Deutschland

\section{The impact of states guidelines on the shape and institutionalisation in processes of region-building}

\begin{abstract}
This article analyzes how states guidelines influence the processes of region-building and how particularly the spatial shape and institutional form of regions can be affected by such governmental guidelines. The nationwide empirical investigation of 1350 program regions in Germany, funded by 27 different programs from five policy fields, leads to two main findings. The first finding of this article is that the spatial shape of regions and their institutional form can be seen as complementary elements of a more stable regional cooperation. The second finding is that the spatial shape and institutional form of regions is significantly influenced by the state's program guidelines.
\end{abstract}

Keywords Regional Governance $\cdot$ Rescaling $\cdot$ Institutionalization $\cdot$ Building of Regions $\cdot$ Regional Cooperations in Germany

\section{Einleitung}

Prozesse der Regionsbildung sind stark beeinflusst vom gewählten oder vorgegebenen Raumzuschnitt einer Region, der Institutionalisierungsform sowie externen Rahmensetzungen. In Deutschland ist eine hohe Spannbreite zu er-

Christian Diller

christian.diller@geogr.uni-giessen.de

1 Institut für Geographie, Justus-Liebig-Universität Gießen, Gießen, Deutschland

2 Institut für Arbeit und Wirtschaft, Universität Bremen, Bremen, Deutschland kennen, sowohl was die territoriale und funktionale $\mathrm{Ab}$ grenzung von Regionen betrifft als auch die zugrundliegenden Regional Governance Formen. Dies kann ein Zusammenschluss von mehreren Landkreisen im Rahmen einer $\mathrm{GmbH}$ wie z.B. in der KielRegion sein, aber auch eine als eingetragener Verein organisierte interkommunale Kooperation, wie die ILE-Region Waginger See-Rupertiwinkel oder ein Regionaler Planungsverband (KöR) wie die Mecklenburgische Seenplatte. Alle diese regionalen Kooperationen unterliegen in einem hohen Maße politisch-rechtlichen Rahmensetzungen, die u. a. in Form von förderpolitischen Anreizen und Vorgaben ihre regionalen Wirkungen auf unterschiedlichen Ebenen entfalten. Diese Formen sind dynamisch sowohl was den räumlichen Zuschnitt als auch 


\begin{tabular}{|c|c|c|c|c|c|}
\hline Politikfeld & Förderprogramm & Laufzeit & Programmregionen & Erfasste Regionel & Fördermittel gesamt \\
\hline \multirow{9}{*}{$\begin{array}{c}\text { Ländliche } \\
\text { Entwicklungs- } \\
\text { politik }\end{array}$} & LEADER I & 1991-1993 & 13 & 13 & ca. 23 Mio. Euro \\
\hline & LEADER II & 1994-1999 & 118 & 116 & ca. 332 Mio. Euro \\
\hline & LEADER + & $2000-2006$ & 148 & 147 & ca. 260 Mio. Euro \\
\hline & LEADER Achse & 2007-2014 & 244 & 237 & ca. 600 Mio. Euro \\
\hline & LEADER ELER & 2015-2020 & 321 & 251 & ca. 1297 Mio. Euro \\
\hline & Regionen Aktiv & 2001-2008 & 18 & 18 & ca. 58,5 Mio. Euro \\
\hline & LandZukunft & 2012-2014 & 9 & 9 & ca. 7,6 Mio. Euro \\
\hline & Land(auf)Schwung & 2015-2018 & 13 & 13 & ca. 19,5 Mio. Euro \\
\hline & Gesamt & & 884 & 804 & \\
\hline \multirow{13}{*}{$\begin{array}{l}\text { Raumordnung } \\
\text { und Landes- } \\
\text { entwicklungs- } \\
\text { politik }\end{array}$} & Regionalkonferenzen & 1995-1996 & 3 & 3 & n.b. \\
\hline & Städtenetze & $1996-2000$ & 12 & 12 & n.b. \\
\hline & Regionen der Zukunft & $1997-2000$ & 26 & 26 & kene Fördernittel \\
\hline & Anpassungsstragien für ländliche Regionen & 2001-2004 & 3 & 3 & n.b. \\
\hline & Innovative Projekte zur Regionalentwicklung 1: Siedlung & 2003-2006 & 9 & 9 & n.b. \\
\hline & Innovative Projekte zur Regionalentwicklung 2: Infrastruktur & 2003-2006 & 6 & 6 & n.b. \\
\hline & kommKOOP & 2005-2006 & 15 & 15 & ca. 30.000 Euro \\
\hline & Regionalplanerische Handlungsansätze zur Daseinsvorsorge & 2007-2009 & 3 & 3 & n.b. \\
\hline & Region schafft Zukunft & 2007-2011 & 4 & 4 & ca. 5,7 Mio. Euro \\
\hline & Aktionsprogramm räumliche Daseinsvorsorge & 2011-2015 & 21 & 21 & ca. 6,5 Mio. Euro \\
\hline & Langfristige Sicherung von Versorgung und Mobilität & 2015-2018 & 18 & 18 & Zwsichen 6,3 und 9 Mio. Euro \\
\hline & Lebendige Regionen & 2015-2022 & 8 & 8 & n.b. \\
\hline & Gesamt & & 128 & 128 & \\
\hline \multirow{4}{*}{$\begin{array}{l}\text { Regionale } \\
\text { Wirtschafts- } \\
\text { poltitik }\end{array}$} & $\begin{array}{l}\text { Gemeinschaftsaufgabe Förderung der regionalen Wirtschaftstruktur (GRW) - Regionales } \\
\text { Entwicklungskonzept }\end{array}$ & seit 1995 & 136 & 135 & n.b. \\
\hline & GRW - Regionales Management & seit 2000 & 45 & 45 & ca. 45,8 Mio. Euro \\
\hline & GRW - Regionalbudget & seit 2008 & 46 & 46 & ca. 33,76 Mio. Euro \\
\hline & Gesamt & & 227 & 226 & \\
\hline \multirow{4}{*}{$\begin{array}{l}\text { Umwelt und } \\
\text { Naturschutz }\end{array}$} & idee.natur & 2007-2020 & 5 & 5 & ca. 36 Mio. Euro \\
\hline & Biosphärenreservate & seit 1979 & 17 & 16 & n.b. \\
\hline & Naturparke & seit 1979 & 102 & 101 & n.b. \\
\hline & Gesamt & & 124 & 122 & \\
\hline \multirow{2}{*}{$\begin{array}{l}\text { Forschung und } \\
\text { Bildung }\end{array}$} & Lernende Regionen & 2002-2008 & 76 & 70 & Ca. 130 Mio. Euro \\
\hline & Gesamt & & 76 & 70 & \\
\hline 5 & 27 & 1991-2016 & 1439 & 1350 & \\
\hline
\end{tabular}

Abb. 1 Erfasste Programmregionen nach Politikfeldern $(N=1350)$. (Quelle: Eigene Darstellung 2021)

die Form der Institutionalisierung angeht, wie dieses Beispiel zeigt: Die schleswig-holsteinische Technologieregion K.E.R.N. entwickelte sich ursprünglich aus einem informellen Städtenetz der Städte Kiel, Eckernförde, Rendsburg und Neumünster. Im Jahr 1991 schloss man sich zu einer flächendeckenden Region (identisch mit dem Planungsraum III des Landes Schleswig-Holstein) zusammen. Diese umfasste vier Kreise und institutionalisierte sich als eingetragener Verein. Ab den frühen 2000er Jahren traten sukzessive die Städte und Landkreise aus. Die Region wurde jedoch 2008 - auch auf Druck der Landesregierung - flächendeckend als KielRegion in Form einer GmbH von drei Kreisen neugegründet.

Eine vertiefte Betrachtung dieser Formen ist sowohl aus praktischer wie theoretischer Sicht interessant. Für die Regionen stellt sich die Frage, ob solche Formen, die das Ergebnis externer Anforderungen der Fördermittelgeber und regionsinterner Akteurs- und Institutionskonstellationen sind, zur Lösung der spezifischen Problemstellungen angemessen sind. Aus Sicht der Forschung ist über diese Frage, aber auch über das Zusammenwirken von Raumzuschnitt und Institutionalisierung, empirisch noch nicht genügend bekannt. Daher werden diese Aspekte werden im Folgenden untersucht. Konkret geht der Beitrag zwei Leitfragen nach:

1. Wie hängen Raumzuschnitt und Institutionalisierungsform regionaler Kooperationen zusammen? Inwieweit hängen lose Raumbezüge mit losen Institutionalisierungen zusammen?

2. Inwieweit gehen strukturell veränderte Raumzuschnitte und Institutionalisierungen in regionalen Kooperationen mit veränderten staatlichen Vorgaben - insbesondere Förderprogrammen - einher bzw. sind durch diese zu erklären?

Wichtig ist der Hinweis, dass mit ,staatlich“ nicht nur nationale, sondern auch supranationale (EU) Vorgaben oder jene von Teilstaaten (Bundesländer) sowie das $\mathrm{Zu}$ sammenwirken von mehreren staatlichen Ebenen gemeint sein können. Programme der EU-Förderung werden z.B. in Deutschland vor allem in den Bundesländern umgesetzt.

Zunächst wird zu diesen Fragen der Stand der Forschung dargelegt. Danach werden die Ergebnisse einer bundesweiten Untersuchung vorgestellt.

\section{Stand der Forschung}

In einem ersten Schritt wird der Stand der Forschung zu den hier wichtigsten Aspekten dargestellt: Raumzuschnitt, Institutionalisierung und die Rolle staatlicher Rahmenvorgaben. 
Abb. 2 Anzahl der geförderten Regionen nach Politikfeld und Regionsabgrenzungsprinzip ( $N=1350$, nur Einfachnennungen möglich). (Quelle: Eigene Untersuchung 2021)

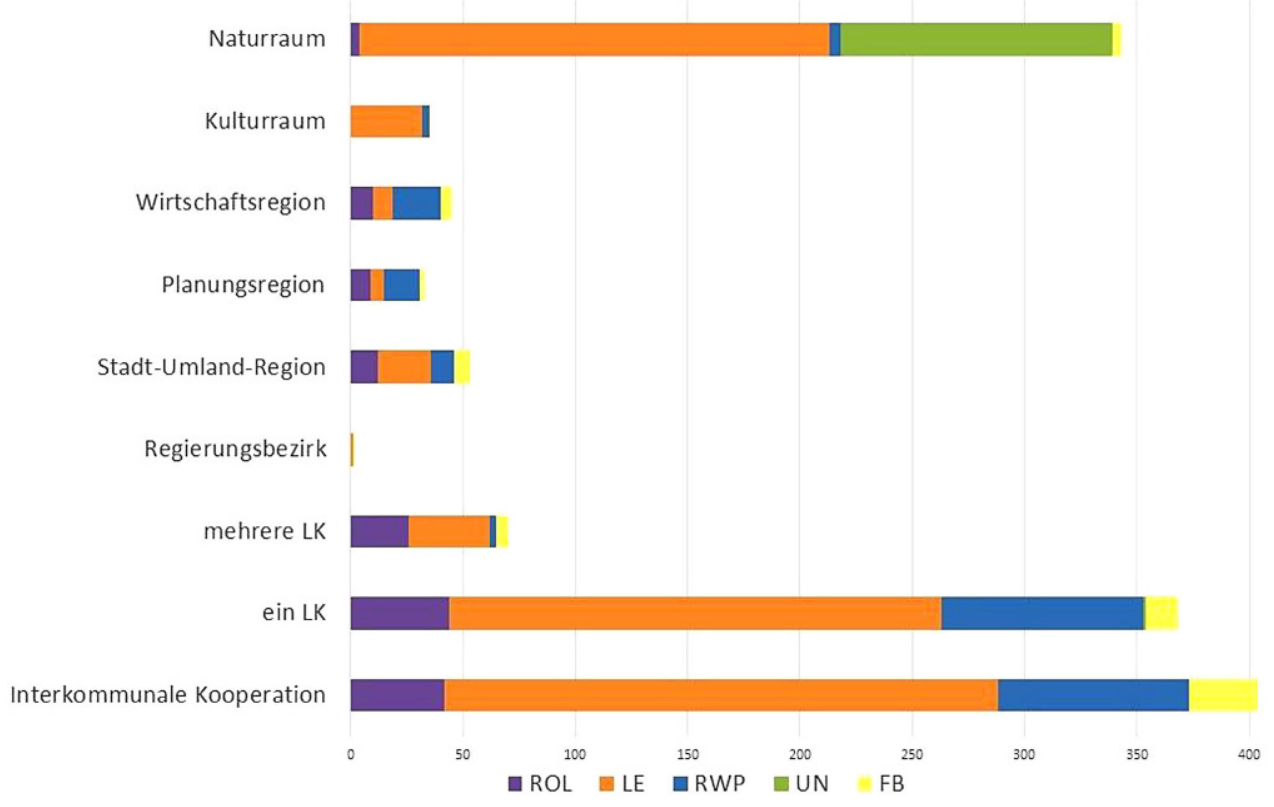

Abb. 3 Anzahl der geförderten Regionen nach Politikfeld und Rechtsform $(N=1287$, nur Einfachnennungen möglich). (Quelle: Eigene Untersuchung 2021)

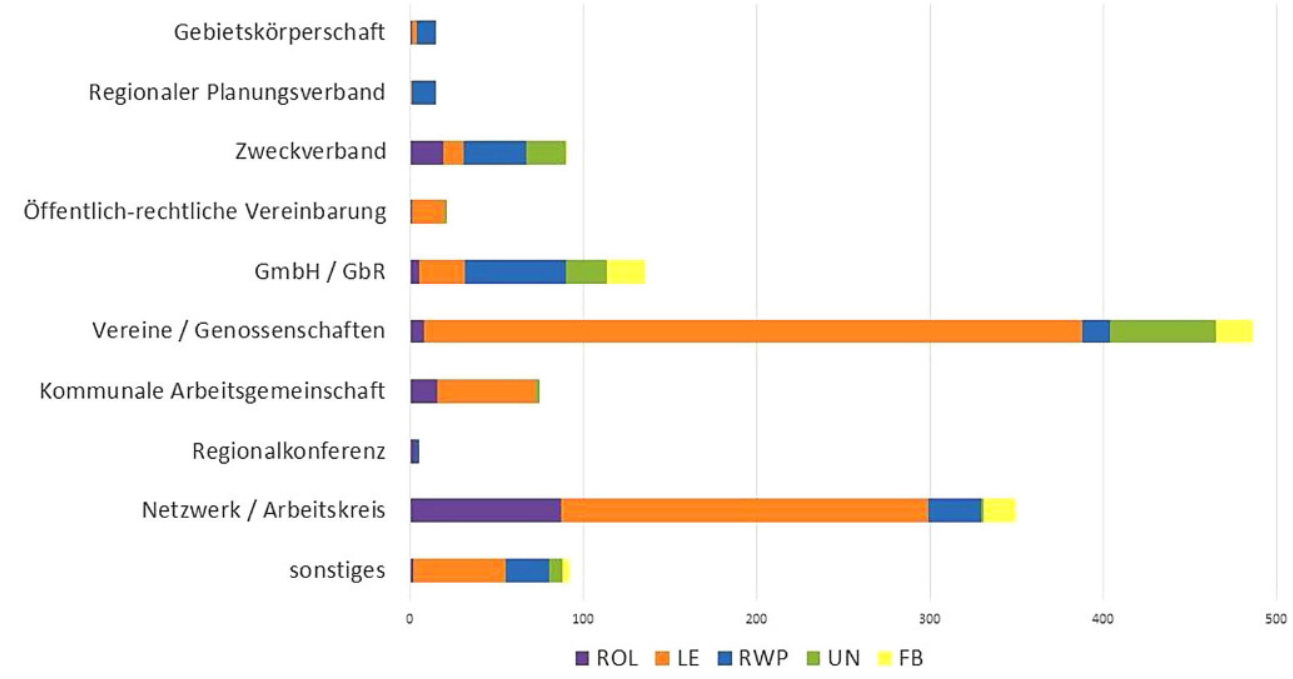

\section{Raumzuschnitt und Institutionalisierung}

Die Debatte um Raumzuschnitte wurde in der Geographie und Soziologie in den USA und Großbritannien seit den 1980er-Jahren und etwas später auch in Deutschland intensiv unter dem Begriff Rescaling geführt (Brenner 1998, S. 432, 2009, S. 124). Territoriale und funktionale Raumbezüge überlappen sich dabei zwar, sind aber nicht vollständig deckungsgleich. Die Trägheit administrativ fest abgegrenzter Einheiten erfordert es, dass politisch-gesellschaftliche Handlungen nicht nur territorial, sondern auch funktional und damit räumlich variabel ausgerichtet sind. Erfolgte eine klassische Regionalisierung eher nach dem Territorialprinzip, in dem erst die Institutionen, dann die Handlungsräume und danach die Diskurse entstanden, so geht moderne
Regionalisierung den umgekehrten Weg: Aus Raumdiskursen und „Proto-Governance“ (Christmann 2010; Gailing 2012; Gailing und Kilper 2010; Matern 2013) entstehen allmählich Handlungsräume. Erst am Ende der Regionsbildungsprozesse stehen eventuell Veränderungen der Institutionen (Reimer 2012, S. 92), die dann dem neuen Scaling angepasst werden. Dadurch überlagern sich häufig in einem Raum unterschiedliche Typen von Mehrebenen-Governance-Strukturen: Typ I umfasst die fest institutionalisierten, räumlich invariablen und nicht überlappenden Formen; Typ II dagegen aufgabenbezogene, räumlich variable und schwach institutionalisierte Einheiten (Gualini 2006, S. 71; Hooghe und Marks 2001). Die Frage des Verhältnisses der Regional Governance-Typen I und II war in Deutschland vor allem bei der Weiterentwicklung der klassischen Re- 
Abb. 4 Anzahl der geförderten Regionen nach Regionsabgrenzungsprinzip und Rechtsform in ausgewählten Politikfeldern (nur Einfachnennungen möglich). (Quelle: Eigene Darstellung 2021)

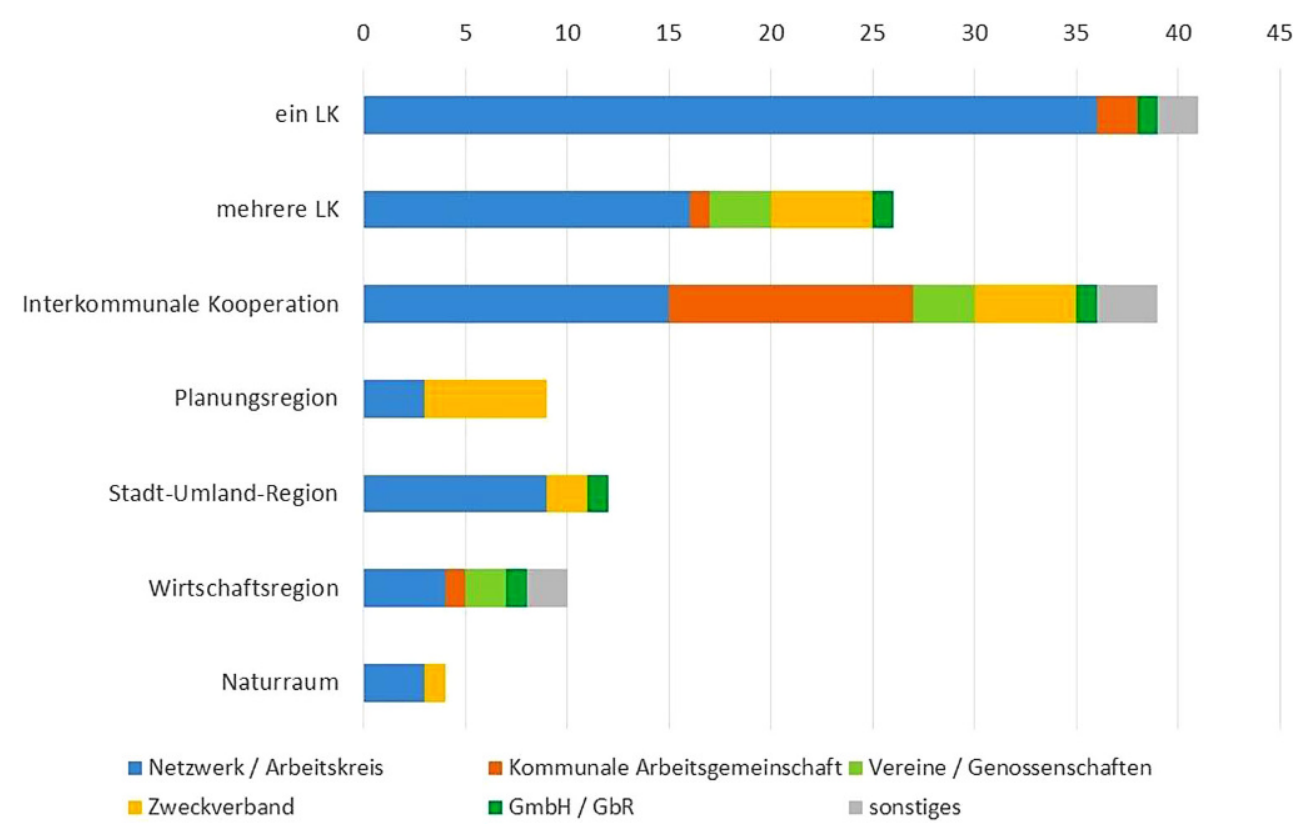

Sie sind jedoch festeren Institutionen unterlegen, wenn es um Routineaufgaben geht oder wenn konflikthafte Entscheidungen getroffen werden müssen. Dann haben lose Netzwerke ein Legitimationsdefizit (Benz und Fürst 2003, S. 205; Diller 2002; Fürst 2006, S. 50). Eine festere Institutionalisierung ist jedoch kein zwangsläufiges Ergebnis der Entwicklung regionaler Kooperationen. Festere institutionelle Strukturen werden zwar oft diskutiert, aber nur selten politisch beschlossen und umgesetzt (Glietsch 2011, S. 86).

\section{Die Rolle staatlicher Vorgaben für die Regionsbildung}

Wenngleich Untersuchungen (z. B. Fürst et al. 2006) zur Regionsbildung die Rolle der staatlichen Vorgaben zwar mit betrachten, gibt es jedoch in Deutschland keine empirische Untersuchung, die hierzu typisierende oder gar generalisierende Aussagen ermöglicht. In den meisten theoretischen Überlegungen zu Regional Governance wird die Rolle des Staates vor allem in der Initiierungsphase regionaler Kooperation gesehen. Es wird dabei häufig unterstellt bzw. die Hoffnung formuliert, dass diese regionalen Kooperationen nach einer Anschubförderung eine Eigendynamik entwickeln und von staatlichen Anreizen immer weiter unabhängig werden. Praktisch ist die Beobachtung jedoch eine andere: Auch im weiteren Verlauf der Kooperation bilden sich meist keine verstetigten horizontalen Selbststeuerungsstrukturen heraus, sondern es sind auch immer wieder Topdown-Impulse erforderlich. Wie sich allerdings diese Topdown-Impulse z. B. zwischen den Politikfeldern, in denen regionale Kooperationen gefördert werden, unterscheiden um Innovationen und Win-Win-Lösungen zu entwickeln. 
und welche Auswirkungen sie haben, darüber ist nur wenig bekannt.

\section{Ergebnisse einer bundesweiten Erhebung regionaler Kooperationen aus 27 Förderprogrammen 1991-2016}

\section{Datengrundlage}

Die empirischen Untersuchungen wurden im Rahmen eines von der DFG geförderten Forschungsprojekts „Entwicklung eines Modells zur Analyse von Lebenszyklen regionaler Kooperationen im Multilevel-Regional-Governance“" (Laufzeit 2015-2017) durchgeführt (Nischwitz et al. 2020). Erfasst wurden die Regionen, die im Zeitraum 1991 bis 2016 im Rahmen der in Abb. 1 dargestellten 27 Förderprogramme gefördert wurden. Insgesamt konnten für 1350 Programmregionen aus fünf verschiedenen Politikfeldern weitergehende Information erfasst und in eine Programmdatenbank eingebettet werden. Den deutlich höchsten Anteil haben hierbei die Regionen, die im Politikfeld Ländliche Entwicklung aus dem LEADER-Programm gefördert wurden. Weitere Politikfelder sind Regionale Wirtschaftspolitik, Raumordnungs- und Landesentwicklungspolitik (ROL), Umwelt und Naturschutz sowie Forschung und Bildung.

Mit dem Ansatz einer politikfeldübergreifenden flächendeckenden Erfassung regionaler Kooperationen ermöglicht diese Datenbank - in einem für Deutschland bislang ungeahntem Ausmaß - statistisch gesicherte Aussagen zu Fragen von Raumzuschnitt, Institutionalisierung und zur Rolle staatlicher Vorgaben.

\section{Ergebnisse}

Regionsabgrenzungen und beispielhafte Rechtsformen der Förderregionen in den einzelnen Politikfeldern

In Konkretisierung grundsätzlicher vorliegender Typologien von Raumzuschnitten (Chilla et al. 2016) wurden hier funktionale Abgrenzungen (Naturraum, Kulturraum) von administrativen Abgrenzungen (Regierungsbezirk, Landkreis, interkommunal) und administrativ funktionalen Mischformen (Planungsregion, Stadt-Umland-Region, Wirtschaftsraum) unterschieden. Die Einordnung der Regionen erfolgte nach dem erkennbaren, dominierenden Schwerpunktprinzip der Raumabgrenzung zum aktuellen Zeitpunkt der Erhebung; Mehrfachnennungen sind also nicht möglich.

Aus Abb. 2 wird deutlich, dass der Großteil (ca. 2/3) der geförderten Regionen sich eng an administrativen Grenzen orientiert. Bei fast einem Drittel handelt es sich um interkommunale Kooperationen. In immerhin einem Vier- tel der Regionen ist der Landkreis mit der Region identisch. Dieses Muster gilt grundsätzlich für alle Politikfelder. Die dennoch signifikanten Unterschiede (Pearson $\mathrm{C}=0,56)^{1}$ zwischen den Politikfeldern erklären sich vor allem durch die abweichende Bedeutung der unterschiedlichen funktionalen Abgrenzungen. Bei den Programmen aus dem Politikfeld Ländliche Entwicklung haben die Regionen, die nach Natur- und Kulturräumen abgegrenzt sind, hohe Bedeutung. Bei den Programmen aus dem Politikfeld Umwelt und Naturschutz wird ausschließlich nach Naturräumen abgegrenzt. Geringer als vermutet ist hingegen bei den Programmregionen aus dem Politikfeld Regionale Wirtschaftspolitik die Bedeutung funktional abgegrenzter Wirtschaftsregionen.

Abb. 3 zeigt die Zusammenhänge zwischen den Politikfeldern und den Rechtsformen der regionalen Kooperation, die Unterschiede sind signifikant (Pearson $\mathrm{C}=0,59$ ). Die insgesamt dominierende Institutionalisierungsform ist die ,mittelharte“ Form des Vereins (meist als eingetragener Verein). Sie ist vor allem bei den Kooperationen aus dem Politikfeld Ländliche Entwicklung stark vertreten, auch die loseren, netzwerkartigen Formen sind in diesem Politikfeld häufig anzutreffen.

Besonders auffallend ist, dass die aus dem Politikfeld ROL geförderten Kooperationen häufig die ,weichsten“ Rechtsformen wie Netzwerk/Arbeitskreis und kommunale Arbeitsgemeinschaften aufweisen. Dies ist ein Hinweis darauf, dass aus diesem Politikfeld besonders oft innovative Ansätze gefördert werden, seltener jedoch die Umsetzung etwa von Infrastrukturmaßnahmen.

Bei den Kooperationen aus dem Politikfeld Regionale Wirtschaftspolitik sind die ,härteren“ privatrechtlichen Formen $\mathrm{GmbH}$ und GbR relativ stark vertreten, wie auch die „harte“ Rechtsform des Zweckverbandes (z. B. Breitbandausbau, Wasserversorgung, Verkehr): Ein Hinweis darauf, dass es hier u. U. um größere Finanzmittel und die Umsetzung investiver Infrastrukturmaßnahmen geht. Auch im Politikfeld Umwelt und Naturschutz finden sich neben dem eingetragenen Verein ebenfalls „härtere“ Formen wie die GbR und der Zweckverband. Das Politikfeld mit den jeweiligen Inhalten bestimmt also die Rechtsform der regionalen Kooperation bereits maßgeblich vor.

\section{Regionsabgrenzung und Rechtsform}

Die Frage nach dem Zusammenhang zwischen Regionsabgrenzung und Rechtsform wird nun für die drei Politikfelder Raumordnung/Landesentwicklung, Ländliche Ent-

\footnotetext{
${ }^{1}$ Der Korrelationskoeffizient Pearson $\mathrm{C}$ misst Zusammenhänge auf nominalem Messniveau und hat eine Spannbreite von 0 bis 1. Ein Wert von 0,56 zeigt eine mittlere bis starke Korrelation an.
} 
wicklung und Raumwirtschaftspolitik differenziert betrachtet (vgl. Abb. 4).

Im Politikfeld Raumordnung/Landesentwicklung besteht eine hohe Korrelation (Pearson C = 0,68) zwischen dem Typ der Regionsabgrenzung und der Rechtsform. Es fällt auf, dass bei einer Regionsabgrenzung, die auf einem Landkreis basiert, die lose Institutionalisierungsform der Netzwerke dominiert - ähnlich wie bei den Stadt-Umland-Regionen und den Naturräumen. Bei interkommunalen Kooperationen ist neben der Form des Netzwerks/Arbeitskreises die Form der kommunalen Arbeitsgemeinschaft relativ stark vertreten. Bei den Wirtschaftsregionen wird neben der Netzwerklösung die Form der Vereinslösung präferiert. Bei den wenigen Fällen mit Planungsregionen dominiert der Zweckverband. „Härtere“ privatrechtliche Formen sind bei den Wirtschafts-, und Stadt-Umland-Regionen relativ stark vertreten.

Auch im Politikfeld Ländliche Entwicklung ist die Korrelation zwischen der Regionsabgrenzung und der Rechtsform zwar signifikant, aber deutlich niedriger (Pearson $\mathrm{C}=0,37$ ) als im Politikfeld Raumordnung/Landesentwicklung. Dies liegt vor allem daran, dass die insgesamt in diesen Programmen sehr stark vertretene Vereinslösung auch in fast allen räumlichen Zuschnitten die dominante Rechtsform ist. Ausgenommen hiervon sind Kooperationen über mehrere Landkreise, wo Netzwerklösungen noch häufiger gewählt werden. Auch hier findet sich die Zweckverbandslösung beim Planungsverband, die „härteren“ Rechtsformen öffentlich-rechtlicher Vereinbarungen und $\mathrm{GmbH}$ fallen in den Wirtschaftsregionen auf.

Im Politikfeld Raumwirtschaftspolitik ist, die Korrelation zwischen Regionsabgrenzung und Rechtsform ebenso hoch (Pearson $\mathrm{C}=0,68$ ) wie im Politikfeld Raumordnung/ Landesentwicklung. Hier fällt auf, dass die für dieses Politikfeld spezifische, ,harte“ privatrechtliche Rechtsform der $\mathrm{GmbH}$ in fast allen Raumzuschnitten - besonders bei den Konstruktionen über mehrere Landkreise - relativ stark vertreten ist. Zweckverbände finden sich besonders häufig bei interkommunalen Kooperationen, Ein-Landkreis-Kooperationen und in Planungsregionen. Interessant ist auch, dass in diesem Politikfeld Regionale Planungsverbände, die die Zusammenarbeit einiger Kommunen in der Region in dem Projekt organisieren, generell stärker vertreten sind als im Politikfeld Raumordnung/Landesentwicklung.

\section{Fazit, Diskussion und weitere Forschungsperspektiven}

In diesem Artikel konnte keine pauschale Antwort auf die für die Regionen wichtige, praktische Frage nach dem zur Bewältigung der jeweiligen Problemstellung erforderlichen Raumzuschnitt und der richtigen Institutionalisierungsform gegeben werden. Diese Frage muss ohnehin im Einzelfall beantwortet werden. Gleichwohl konnten einige RegelmäBigkeiten herausgearbeitet werden.

Aus den Analysen ergeben sich zwar keine generalisierbaren Handlungsempfehlungen an Regionen, wie Raumzuschnitt und Institutionalisierungsform miteinander in Einklang gebracht werden können. $\mathrm{Zu}$ stark variieren hier vor allem die Akteurskonstellationen vor Ort - jeder Fall ist anders. Jedoch ergaben sich Anhaltspunkte für folgende Komplementaritätsthese: Feste organisatorische Institutionalisierung und eine Regionsabgrenzung, die an festen administrativen Grenzen orientiert ist, müssen nicht einhergehen; im Gegenteil: Sie ergänzen sich. Die Risiken einer nur losen netzwerkartigen Institutionalisierung der Akteursbeziehungen werden abgemildert, wenn die Kooperation im etablierten Raumzuschnitt eines Landkreises stattfindet. Die Kooperation erfolgt zwar nicht primär innerhalb der Institutionen des Landkreises, sie basiert jedoch auf diesen bestehenden Strukturen. Die empirischen Befunde zur Frage der Rolle staatlicher Vorgaben zeigen, dass es sich bei Regional Governance nicht nur um horizontale Selbstkoordination handelt (Diller 2016a). Auch und gerade wenn im Sinne des Regional-Governance-Begriffs zunehmend Akteure aus Wirtschaft und Zivilgesellschaft einbezogen werden, so wird doch die Form regionaler Kooperationen ganz maßgeblich von den staatlichen Rahmensetzungen beeinflusst. Dies machen bereits die systematischen Unterschiede zwischen den einzelnen Politikfeldern deutlich, was die Themen- und Akteursanzahl, den räumlichen Zuschnitt und die Institutionalisierung und damit zusammenhängend den „Härtegrad“ der Aufgabenstellungen (Innovation vs. Umsetzung) angeht. Weiterhin sind innerhalb der Politikfelder auch unterschiedlich enge Zusammenhänge zwischen Regionsabgrenzung und Rechtsform feststellbar. Bei der Frage 2 des Einflusses von staatlichen Rahmensetzungen ist ein Zusammenhang zu den staatlichen Vorgaben und Regionsabgrenzung bzw. Rechtsform zu erkennen. Dabei gilt: Form follows function. Wenn, wie in den von der Bundesraumordnung initiierten Programmen, eher Innovation gewünscht wird, ist z.B. die „Rechtsform“ loser Netzwerke besonders oft vertreten. Sollen dagegen regionale Entwicklungsprozesse mit einem breiten Akteurskreis umgesetzt werden, wie in den LEADER-Programmen, scheint der Verein eine angemessene institutionelle Lösung. Oder es kommen vor allem in den Programmen zur regionalen Wirtschaftsentwicklung auch „härtere“ privatrechtliche Lösungen zum Tragen. Inwieweit dieser beobachtete statistische Zusammenhang tatsächlich als signifikanter Kausalzusammenhang bezeichnet werden kann, wäre Gegenstand weiterer Forschung, insbesondere durch einen Vergleich von geförderten und nicht geförderten Regionen unter Einbeziehung von Kontrollvariablen. Die weitere Forschung sollte sich weiterhin vor allem auf die durch sich 
wandelnde staatliche Rahmenvorgaben erforderlichen Anpassungsleistungen der Regionen richten. Hierbei können auch die jüngeren Ansätze der Analyse kognitiver Prozesse der Regionsbildung (vgl. u. a.: Zimmermann 2012) fruchtbar gemacht werden. Die Frage des Warums von Regionszuschnitt und Institutionalisierung, zu der diese quantitative Untersuchung erste Zusammenhänger ermittelte, kann mit Fallstudienanalysen vertieft herausgearbeitet werden.

Funding Open Access funding enabled and organized by Projekt DEAL.

Open Access Dieser Artikel wird unter der Creative Commons Namensnennung 4.0 International Lizenz veröffentlicht, welche die Nutzung, Vervielfältigung, Bearbeitung, Verbreitung und Wiedergabe in jeglichem Medium und Format erlaubt, sofern Sie den/die ursprünglichen Autor(en) und die Quelle ordnungsgemäß nennen, einen Link zur Creative Commons Lizenz beifügen und angeben, ob Änderungen vorgenommen wurden.

Die in diesem Artikel enthaltenen Bilder und sonstiges Drittmaterial unterliegen ebenfalls der genannten Creative Commons Lizenz, sofern sich aus der Abbildungslegende nichts anderes ergibt. Sofern das betreffende Material nicht unter der genannten Creative Commons Lizenz steht und die betreffende Handlung nicht nach gesetzlichen Vorschriften erlaubt ist, ist für die oben aufgeführten Weiterverwendungen des Materials die Einwilligung des jeweiligen Rechteinhabers einzuholen.

Weitere Details zur Lizenz entnehmen Sie bitte der Lizenzinformation auf http://creativecommons.org/licenses/by/4.0/deed.de.

\section{Literatur}

Allmendinger P, Chilla T, Sielker F (2014) Europeanizing territoriality—towards soft spaces? Environ Plan A 46(11):2703-2717. https://doi.org/10.1068/a130037p

Benz A, Fürst D (2003) Region, Regional Governance, Regionalentwicklung. In: Adamaschek B, Pröhl M (Hrsg) Regionen erfolgreich steuern. Regional Governance - von der kommunalen zur regionalen Strategie. Bertelsmann, Gütersloh, S 11-66

Brenner N (1998) Between fixity and motion: accumulation, territorial organization and the historical geography of spatial scales. Environ Plan D 16(4):459-481. https://doi.org/10.1068/d160459

Brenner N (2009) Ten questions on state rescaling. Camb J Reg Econ Soc 2(1):123-129. https://doi.org/10.1093/cjres/rsp002

Chilla T, Kühne O, Neufeld M (2016) Regionalentwicklung. UTB,

Christmann GB (2010) Kommunikative Raumkonstruktionen als (Proto-) Governance. In: Kilper H (Hrsg) Governance und Raum. Nomos, Baden-Baden, S 27-48

Diller C (2002) Zwischen Netzwerk und Institution: eine Bilanz regionaler Kooperationen in Deutschland. Opladen,
Diller C (2016a) Die „Zweite Generation“: Zum Stand und zu den Perspektiven der theoriebasierten Regional Governance-Forschung in Deutschland. DisP Plan Rev 206(3):16-31. https://doi.org/10. $1080 / 02513625.2016 .1235872$

Diller C (2016b) Development of metropolitan regions in Germany in light of the restructuring of the German states. Eur Plan Stud 24(12):2154-2174. https://doi.org/10.1080/09654313.2016. 1258040

Fürst D (1994) Regionalkonferenzen zwischen offenen Netzwerken und fester Institutionalisierung. Raumforsch Raumordn 52(3):184-192

Fürst D (2006) Regional Governance, ein Überblick. In: Kleinfeld R, Plamper H, Huber A (Hrsg) Regional Governance, Bd. 1. V\&R unipress, Göttingen, S 35-60

Fürst D, Lahner M, Pollermann K (2006) Entstehung und Funktionsweise von Regional Governance bei dem Gemeinschaftsgut Natur und Landschaft. Analyse von Place-Making- und GovernanceProzessen in Biosphärenreservaten in Deutschland und Großbritannien (Hannover)

Gailing L (2012) Sektorale Institutionensysteme und die Governance kulturlandschaftlicher Handlungsräume. Eine institutionen- und steuerungstheoretische Perspektive auf die Konstruktion von Kulturlandschaft. Raumforsch Raumordn 70:147-160. https://doi. org/10.1007/s13147-011-0135-x

Gailing L, Kilper H (2010) Institutionen und Handlungsräume als sozio-politische Konstruktionen. In: Kilper H (Hrsg) Governance und Raum. Nomos, Baden-Baden, S 93-110

Glietsch C (2011) Regional Governance in stark institutionalisierten Strukturen. Eine Studie zur Innovationsfähigkeit ausgewählter Metropolregionen, Hamburg

Gualini E (2006) Mehrebenen-Governance und neue Formen von Territorialität. Institutioneller Wandel in der Regionalpolitik. In: Kleinfeld R, Plamper H, Huber A (Hrsg) Regional Governance, Bd. 1. V\&R unipress, Göttingen, S 61-82

Hooghe L, Marks G (2001) Eur Integr Online Pap 5:11

Matern A (2013) Mehrwert Metropolregion. Stadt-Land-Partnerschaften und Praktiken der Raumkonstruktion in der Metropolregion Hamburg. Transcript, Bielefeld

Nischwitz G, Diller C, Kohl M, Chojnowski P (2020) Entwicklung eines neuen Modells zur Analyse regionaler Governanceprozesse. DisP Plan Rev 56(2):32-50. https://doi.org/10.1080/02513625. 2020.1794125

Reimer M (2012) Planungskultur im Wandel. Das Beispiel der Regionale 2010. Rohn, Dortmund

Sielker F, Chilla T (2015) Regionen als ,Soft Spaces“? Das neue EUInstrument der makroregionalen Strategien. In: Kühne O, Weber F (Hrsg) Bausteine der Regionalentwicklung. RaumFragen: Stadt Region - Landschaft (Stadt -Region - Landschaft). Springer VS, Wiesbaden, S 41-54

Walsh C, Jacuniak-Suda M, Knieling J, Othengrafen F (2012) Soft Spaces in spatial planning and governance: theoretical reflections and definitional issues. Conference Paper HCU, Hamburg

Zimmermann K (2012) Institutionalisierung regionaler Kooperation als kollektiver Lernprozesse. Das Beispiel der Metropolregion Rhein-Neckar. Nomos, Baden-Baden 